\title{
Attempts to improve human ovarian transplantation outcomes of needle-immersed vitrification and slow-freezing by host and graft treatments
}

\author{
Ronit Abir $^{1,2}$ • Benjamin Fisch ${ }^{1,2}$ - Noa Fisher ${ }^{1,2}$ - Nivin Samara ${ }^{1,2,3}$. \\ Galit Lerer-Serfaty $^{1,2} \cdot$ Roei Magen ${ }^{1,4}$ • Michal Herman-Edelstein ${ }^{5}$. \\ Avi Ben-Haroush ${ }^{1,2} \cdot$ Anat Stein $^{1,2} \cdot$ Raoul Orvieto ${ }^{2,6}$
}

Received: 22 August 2016 / Accepted: 24 January 2017 /Published online: 18 March 2017

(C) Springer Science+Business Media New York 2017

\begin{abstract}
Purpose To investigate if needle-immersed vitrification or slow-freezing yields better implantation results for human ovarian tissue and which method benefits more when combined with the "improvement protocol" of host melatonin treatment and graft incubation with biological glue + vitamin $\mathrm{E}+$ vascular endothelial growth factor-A.

Methods Human ovarian tissue was preserved by needleimmersed vitrification or slow-freezing and transplanted into immunodeficient mice, either untreated (groups $\mathrm{A}$ and $\mathrm{C}$, respectively) or treated with the improvement protocol (groups B and D, respectively). Grafted and ungrafted slices were evaluated by
\end{abstract}

Dr. Noa Fisher D.M.D. performed most of the experiments as a partial requirement for her M.Sc. degree at Sackler Faculty of Medicine, Tel Aviv University, Tel Aviv, Israel.

Ronit Abir

ronita@clalit.org.il

1 Infertility and IVF Unit, Beilinson Women Hospital, Rabin Medical Center, Petach Tikva 49100, Israel

2 Sackler Faculty of Medicine, Tel Aviv University, Tel Aviv 69978, Israel

3 Present address: In Vitro Fertilization Unit, Lis Maternity and Women's Hospital, Tel Aviv Sourasky Medical Center, 6423906 Tel Aviv, Israel

4 Faculty of Health Sciences, Goldman Medical School, Ben Gurion University of the Negev, Beer Sheva 8410501, Israel

5 Department of Nephrology, Rabin Medical Center, Felsenstein Research Center 49100 and Sackler Faculty of Medicine, Tel Aviv University, Tel Aviv 69978, Israel

6 Infertility and IVF Unit, Department of Obstetrics and Gynecology, The Chaim Sheba Medical Center, Ramat Gan 52621, Israel follicle counts, apoptosis assay and immunohistochemistry for Ki67 and platelet endothelial cell adhesion molecule (PECAM).

Results Follicle number in the recovered grafts was limited. The number of atretic follicles was significantly higher after vitrification with/without the improvement protocol and slowfreezing than that after slow-freezing + the improvement protocol. Stroma cell apoptosis was the lowest in the group D. PECAM staining showed a peripheral and diffuse pattern in the group D (mostly normal follicular morphology) and a diffuse pattern in all other groups (few follicles, mostly atretic), with significantly higher diffuse levels in the vitrification groups. Ki67 staining was identified in all normal follicles. Follicles did not survive transplantation in the vitrification groups.

Conclusions Ovarian sample preparation with slow-freezing + the improvement protocol appears to yield better implantation outcomes than needle-immersed vitrification with/ without the improvement protocol. The real quality of frozen tissue can be assessed only after grafting and not after thawing/warming.

Keywords Human ovarian tissue $\cdot$ Needle-immersed vitrification · Slow-gradual freezing . "Improvement protocol” · PECAM $\cdot$ Ki67 $\cdot$ TUNEL

\section{Introduction}

With the increase in cancer survival rates in young women of reproductive age, more attention is being addressed to the management of radiation- and chemotherapy-related ovarian failure [1]. Among the limited options available for fertility preservation is cryopreservation of ovarian cortical tissue containing immature primordial follicles followed by autotransplantation. 
This procedure has so far resulted in over 70 livebirths [2], most of which were achieved after slow-gradual freezing/thawing. However, three successful deliveries have been reported after vitrification followed by in vitro activation [3, 4].

Although vitrification has been suggested as a potential alternative to slow-freezing [5], studies of human ovarian tissue have so far yielded conflicting results. Some reported the same results for both methods or better results for slowfreezing than vitrification, whereas others found that vitrification was associated with increased stroma cell survival [6-9] compared to slow-freezing, despite their similar follicular viability. In monkeys, vitrification was shown to be successful, with promotion of follicular development [10], especially when the tissue was implanted into freshly decorticated vascular beds [11]. Researchers have introduced a method of needle-immersed vitrification wherein thinly sliced ovarian tissue is placed on acupuncture needles and transferred to the vitrification solution $[6,9]$. This method is not only practical and convenient but also produced excellent results for human ovarian tissue in terms of follicular and stroma cell survival.

A major obstacle in ovarian tissue transplantation is follicular loss immediately after grafting, possibly due to slow neovascularization that leads to ischemia $[12,13]$. Alternatively, post-transplantation follicular loss, might have been a result of primordial follicle "burn-out", as demonstrated for thinly sliced grafted bovine ovarian tissue [14], similar to that used for vitrification.

Melatonin and vitamin E are free radical scavengers with a broad anti-oxidant activity spectrum and antiapoptotic function $[12,13]$. Vascular endothelial growth factor-A (VEGF-A) is the most potent regulator of blood vessel formation. Hyaluronan (HA) is an important component of the extracellular matrix in the reproductive tract, and promotes anti-inflammatory and anti-apoptotic signals [13]. Studies in immunodeficient mice showed better outcomes when slow-frozen human ovarian samples were grafted after treatment of the host with melatonin and pretransplant incubation of the graft with HA-rich biological glue + vitamin E + VEGF-A, termed the "improvement protocol" [13].

So far, the improvement protocol has not been applied to vitrified-warmed human ovarian tissue. However, researchers have suggested that given the improvement in stroma cell quality associated with vitrification, including the needle-immersion method, the use of vitrified/warmed ovarian tissue might enhance graft neovascularization [6-9].

The aims of the present study were to compare the outcomes of needle-immersed vitrification and slowfreezing for human ovarian tissue grafting and to determine which method benefits more from combination with the improvement protocol.

\section{Material and methods}

\section{Source of ovarian tissue}

The study was approved by the local institutional ethics committee. Tissue was obtained from nine girls/women aged 1331 years (mean age \pm standard deviation (SD) $21 \pm 6$ years) during laparoscopic ovarian surgery for fertility cryopreservation before anticancer therapy (Tables 1 and 2) [1]. None of the patients were prepubertal. Informed consent to donate tissue for the present study was obtained from the patients or the parents of minors. One slice measuring 1-2 $\mathrm{mm}$ (with a depth of 1-2 mm) from every ovarian sample was fixed in Bouin's solution (prepared from compounds purchased from Sigma, St Louis, MO, USA) immediately after ovarian dissection (fresh ungrafted control) to evaluate pre-grafting follicular density. This procedure was done to ensure, to the extent possible, use of follicle-rich samples. Our long experience with human ovarian tissue suggests that pre-grafting or preculturing evaluation of follicular density increases the chances of utilizing slices with ovarian follicles [12, 13, 15]. Moreover, fertility restoration will be conducted with frozen tissue and not fresh tissue.

\section{Slow-freezing-thawing of ovarian tissue}

Cryopreservation was carried out within $1 \mathrm{~h}$ of ovarian retrieval. Our slow-freezing-thawing procedure has been described in detail previously $[12,13]$. In brief, all the ovarian samples were cut into slices $1 \times 0.5 \mathrm{~cm}$ (with a depth of $1-2 \mathrm{~mm}$ ), and the slices were placed in cryogenic vials (Nalge Nunc International, Delta, Roskilde, Denmark) filled with $1.5 \mathrm{M}$ dimethylsulfoxide (DMSO, Sigma). Prior to freezing, the samples were kept on ice for $30 \mathrm{~min}$ to establish equilibration. They were then frozen slowly in a programmable freezer (Kryo 10; series 10/20, Planer Biomed, Sunbury on Thames, UK) and immediately placed in liquid nitrogen. Thawing was carried out by washouts with decreasing concentration gradients of DMSO (Sigma). One slow-frozen/thawed slice from every ovarian sample (similar in dimensions to the fresh control) was fixed in the Bouin's solution immediately after ovarian thawing (slow frozen-thawed ungrafted control).

\section{Needle-immersed vitrification/warming of human ovarian tissue (Fig. 1)}

Cortical slices were cut extra thin (dimension $\sim 1 \times 0.5 \mathrm{~cm}$; depth $\sim 0.5 \mathrm{~mm}$ ) $[7,15,16]$ and placed on insulin needles connected to 1-ml syringes (Tuberkulin, Henke Sass Wolf, Tuttingen, Germany) (Fig. 1). Our laboratory is experienced with this delicate sectioning of human ovarian tissue [15]. All solutions were diluted with phosphate-buffered saline (PBS; 
Table 1 Ovarian transplantation after vitrification

\begin{tabular}{|c|c|c|c|c|c|c|c|c|}
\hline \multirow[b]{2}{*}{ Pt. no. } & \multirow[b]{2}{*}{ Age (year) } & \multirow[b]{2}{*}{ Hosts $(n)$} & \multicolumn{2}{|c|}{$\begin{array}{l}\text { Follicles, fresh } \\
\text { ungrafted }\end{array}$} & \multicolumn{2}{|c|}{$\begin{array}{l}\text { Follicles, vitrified } \\
\text { ungrafted }\end{array}$} & \multicolumn{2}{|c|}{$\begin{array}{l}\text { Follicles, after } \\
\text { grafting }\end{array}$} \\
\hline & & & Preantral & Atretic & Preantral & Atretic & Preantral & Atretic \\
\hline \multicolumn{9}{|c|}{ A. Vitrification only } \\
\hline 1 & 13 & 2 & 4 & & 16 & 0 & 0 & 0 \\
\hline 2 & 16 & 3 & 25 & 0 & - & - & 7 & 3 \\
\hline 3 & 18 & 1 & 5 & 0 & 28 & 2 & 2 & 1 \\
\hline 4 & 18 & 5 & 34 & 0 & 24 & 0 & 28 & 0 \\
\hline 5 & 19 & 2 & 25 & 0 & 23 & 0 & 34 & 34 \\
\hline 6 & 21 & 6 & 2 & 0 & 1 & $\mathbf{0}$ & 24 & 16 \\
\hline 7 & 22 & 4 & 2 & & 1 & $\mathbf{0}$ & 3 & 2 \\
\hline 8 & 29 & 2 & 31 & 1 & 37 & 0 & 0 & 0 \\
\hline 9 & 31 & 3 & 2 & 1 & 2 & 2 & 10 & 10 \\
\hline Tota & & 28 & 128 & $2(2)$ & 132 & $4(3)$ & 88 & $50(57)^{\mathrm{a}}$ \\
\hline \multicolumn{9}{|c|}{ B. Vitrification + improvement protocol } \\
\hline 1 & 13 & 3 & 4 & 0 & 16 & 0 & 5 & 5 \\
\hline 2 & 16 & 3 & 25 & 0 & 0 & 0 & 7 & 7 \\
\hline 3 & 18 & 2 & 5 & 0 & 28 & 2 & 2 & 0 \\
\hline 4 & 18 & 4 & 34 & 0 & 24 & 0 & 28 & 10 \\
\hline 5 & 19 & 2 & 25 & 1 & 23 & 0 & 34 & 15 \\
\hline 6 & 21 & 4 & 2 & 1 & 1 & 0 & 24 & 14 \\
\hline Tota & & 18 & 95 & $2(2)$ & 92 & $2(2)$ & 95 & $51(54)^{\mathrm{a}}$ \\
\hline
\end{tabular}

Every follicular class is calculated as a percent from total follicles/section per group; - no slide available ${ }^{a}$ Significantly higher than the fresh, vitrified, and slow-frozen ungrafted and group D (Table 2), $p<0.0001$
Table 2 Ovarian transplantation after slow-freezing

\begin{tabular}{|c|c|c|c|c|c|c|c|c|}
\hline \multirow[b]{2}{*}{ Pt. no. } & \multirow[b]{2}{*}{ Age (year) } & \multirow[b]{2}{*}{ Hosts $(n)$} & \multicolumn{2}{|c|}{$\begin{array}{l}\text { Follicles, fresh } \\
\text { ungrafted }\end{array}$} & \multicolumn{2}{|c|}{$\begin{array}{l}\text { Follicles, frozen } \\
\text { ungrafted }\end{array}$} & \multicolumn{2}{|c|}{$\begin{array}{l}\text { Follicles, after } \\
\text { grafting }\end{array}$} \\
\hline & & & Preantral & Atretic & Preantral & Atretic & Preantral & Atretic \\
\hline \multicolumn{9}{|c|}{ C. Slow-freezing only } \\
\hline 1 & 13 & 2 & 4 & 0 & 1 & 0 & 5 & 5 \\
\hline 2 & 16 & 3 & 25 & 0 & 1 & 0 & 0 & 0 \\
\hline 3 & 18 & 3 & 5 & 0 & 5 & 0 & 1 & 1 \\
\hline 4 & 18 & 3 & 34 & 0 & 6 & 0 & 44 & 44 \\
\hline 5 & 19 & 3 & 25 & 1 & 12 & 0 & 6 & 4 \\
\hline 6 & 21 & 3 & 2 & 1 & 7 & 4 & $\mathbf{0}$ & 0 \\
\hline Total $(\%)$ & & 17 & 95 & $2(2)$ & 32 & $4(13)$ & 56 & $54(96)^{\mathrm{a}}$ \\
\hline \multicolumn{9}{|c|}{ D. Slow-freezing + improvement protocol } \\
\hline 1 & 13 & 3 & 4 & 0 & 1 & 0 & 17 & 7 \\
\hline 2 & 16 & 2 & 25 & 0 & 1 & 0 & 6 & 2 \\
\hline 3 & 18 & 3 & 5 & 0 & 5 & 0 & 28 & 0 \\
\hline 4 & 18 & 3 & 34 & 0 & 6 & 0 & 25 & 0 \\
\hline 5 & 19 & 3 & 25 & 1 & 12 & 0 & 10 & 10 \\
\hline 6 & 21 & 4 & 2 & 1 & 7 & 4 & 8 & 2 \\
\hline Total $(\%)$ & & 18 & 95 & $2(2)$ & 32 & $4(13)$ & 94 & $21(22)^{b}$ \\
\hline
\end{tabular}

Every follicular class is calculated as a percent from total follicles/section per group

${ }^{a}$ Significantly higher than the fresh, vitrified, and slow-frozen ungrafted and group $\mathrm{D}, p<0.0001$

${ }^{\mathrm{b}}$ Significantly higher than the fresh and vitrified ungrafted $(p<0.0001)$ and slow-frozen ungrafted $(p=0.03)$ 
Fig. 1 Needle-immersed vitrification. a Thinly sliced human ovarian slices placed on an insulin needle connected to a syringe and dipped in a cryotube containing the equilibration solution. b Thinly sliced human ovarian tissue placed on insulin needles attached to a syringe.
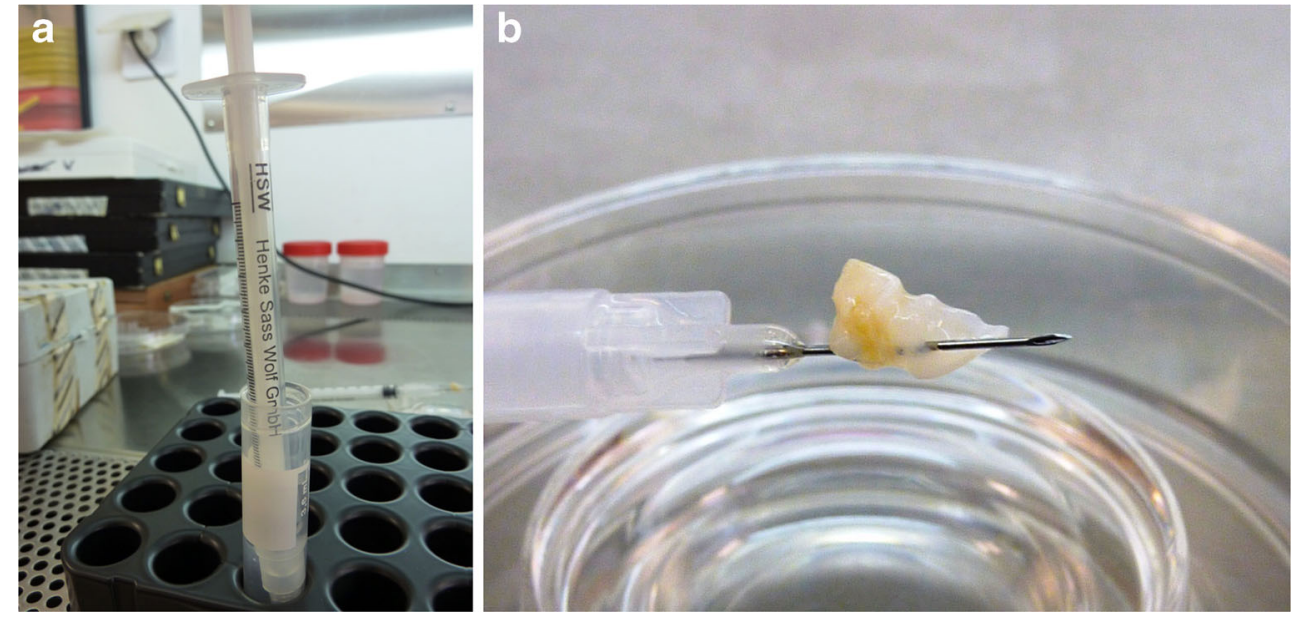

Biological Industries, Beit Ha'emek, Israel). The needles were dipped in succession into two 3-ml cryotubes (Nalge Nunc International) filled with the relevant solution: (1) an equilibration solution containing $7.5 \%$ ethylyne glycol (EG-Sigma), 7.5\% DMSO (Sigma), and 20\% serum substitute supplement (Irvine Scientific, Santa Ana, CA, USA) for 10-min incubation $[6,9]$, and (2) the vitrification solution containing $15 \%$ EG (Sigma), 15\% DMSO (Sigma), and 20\% serum substitute supplement (Irvine Scientific) for 5-min incubation. The needles were then removed from the syringes, the excess vitrification fluid was absorbed using sterile pads, and the needles were rapidly plunged with new 3-ml cryotubes (Nalge Nunc International) into liquid nitrogen.

Warming was performed by rinsing the slices for $5 \mathrm{~min}$ in series of solutions prepared from sucrose (Sigma) as follows: $1 \mathrm{M}$ (prewarmed to $37^{\circ} \mathrm{C}$ ), $0.5 \mathrm{M}(5 \mathrm{~min}$ at room temperature (RT)), and $0.25 \mathrm{M}$ (5 min at RT). The slices were then rinsed with PBS (Biological Industries) with $20 \%$ serum substitute supplement (Irvine Scientific) for $20 \mathrm{~min}$ at RT. One vitrified/ warmed slice from every ovarian sample (similar in dimensions to the fresh control but extra thin with a depth $\sim 0.5 \mathrm{~mm}$ ) was fixed in the Bouin's solution immediately after ovarian warming (vitrified/warmed ungrafted control).

\section{Transplantation into immunodeficient mice}

Eighty-one immunodeficient nu/nu female Balb/C mice aged 10-12 weeks (Harlan, Jerusalem, Israel) were used for tissue transplantation (Tables 1 and 2). The frozen/thawed or vitrified warmed slices were further cut to dimensions of $\sim 3 \times$ $3 \mathrm{~mm}$ (depth $\sim 1 \mathrm{~mm}$ for slow-frozen thawed samples, $\sim 0.5 \mathrm{~mm}$ for vitrified-warmed samples). One ovarian sample was transplanted into every mouse, and the mice were divided into four treatment groups as follows:

(A) grafting with vitrified/warmed ovarian tissue slices without further treatment.
(B) grafting with vitrified/warmed ovarian slices combined with the improvement protocol [13]: melatonin (240 mg/ 1, Sigma) dissolved in the drinking water $(60 \mathrm{mg}$ in a 250 -ml bottle) in a light sealed bottle for 2 weeks prior to grafting and throughout the experiment, and pretransplant graft incubation at $37^{\circ} \mathrm{C}$ for $2 \mathrm{~h}$ with a solution of human recombinant VEGF-A (200 ng/ml, Biological Industries), and vitamin E-oily form (400 IU/ml, E-400 natural, SOFT GEL Technologies Inc, Los Angeles, CA, USA) mixed in HA-rich biological glue (Uterine Transfer Medium, UTM medium, MediCult, Jyllinge, Denmark), to a final volume of $1 \mathrm{ml}$.

(C) grafting with slow-gradual frozen/thawed ovarian tissue without further treatment.

(D) grafting with slow-gradual frozen/thawed ovarian tissue combined with the improvement protocol as in the treatment group B.

Ovarian samples from six of the nine patients were used in parallel for all groups. For the vitrification-only group (group A, Table 1), we also included tissue from three additional patients, who were the oldest in the study (patients 7-9, ages 22, 29, and 31 years). The ungrafted fresh, slow-frozen, and vitrified controls were from the same patients from which tissue was grafted after freezing.

All the mice were anesthetized with an intramuscular injection of $0.04 \mathrm{ml}$ of a $1: 1$ solution of $0.5 \%$ xylazine base (prepared from 2\% XYL-M, VMD, Arendonk, Belgium, diluted with sterile distilled water) and $50 \mathrm{mg} / \mathrm{ml}$ ketamine hydrochloride (Ketalar, Parke Davis, Hampshire, UK) [17]. We chose the back muscle as the transplantation site because it is not richly vascularized, and we assumed it could mimic ovarian conditions. Moreover, our previous studies reported high recovery rates after back muscle transplantation [12, 13]. After the back muscle was dissected, one ovarian slice per mouse was transplanted. The mice were euthanized 3 weeks after surgery, and the transplants were removed and fixed in the 
Bouin's solution (all compounds were purchased from Sigma). We chose 3 weeks, because that was the limit in our previous studies $[12,13]$ and because we wanted to demonstrate events that occur shortly after transplantation: neovascularization and follicle survival rather than follicular development.

\section{Histological preparation}

Control ungrafted and transplanted ovarian tissue specimens were prepared for paraffin embedding, as described by us previously $[12,13]$. The follicles in the fresh and thawed controls and transplanted specimens (Tables 1 and 2) were counted from the hematoxylin and eosin-stained sections with a computerized image analyzer (analySIS, Soft Imaging System, Digital Solutions for Imaging and Microscopy, System GmbH, Munster, Germany). The number of follicles in the uncultured and cultured samples was counted throughout the field (magnification $\times 100$ ) in two levels per specimen, with at least $50 \mu \mathrm{m}$ between levels to avoid counting the same follicle twice. The follicles were defined preantral (before the antral stage): primordial (the oocyte is surrounded by a flat layer of granulosa cells), primary (the oocyte is surrounded by a cuboidal layer of granulosa cells), or secondary (the oocyte is surrounded by at least two granulosa cell layers enclosed by a theca layer) [18]. Atretic follicles were characterized by pyknotic cells, eosinophilia of the ooplasm, and clumping of the chromatin material. Unstained sections were placed on OptiPlus positive-charged microscope slides (BioGenex Laboratories, San Ramon, CA, USA) for apoptosis evaluation and immunohistochemistry studies.

\section{Terminal deoxynucleotidyl transferase assay (TUNEL)}

Apoptosis was evaluated in the thawed control ungrafted specimens and the transplanted specimens using the terminal deoxynucleotidyl transferase (TdT) (TUNEL) assay (ApopTag, In Situ Detection Kit; Intergen Company, Purchase, NY, USA), as described by us previously $[12,13]$. There were two positive controls: the first was provided with the kit and included a section from a rat mammary gland 4 days after weaning, and the second was prepared by exposure of the human ovarian sections to DNAase $(1 \mu \mathrm{g} / \mathrm{ml}$, Sigma) for $15 \mathrm{~min}$. For the negative control, the equilibrium buffer provided with the kit replaced the diluted TdT enzyme. Two samples from each patient in all the study groups underwent the TUNEL procedure and were analyzed.

Stroma cell TUNEL staining intensity was graded on a 4point scale, as described by us previously $[12,13]$ : $0=$ no TUNEL staining, 1 = TUNEL-expressing cells with low staining intensity, $2=$ TUNEL-expressing cells with medium staining intensity, and $3=$ TUNEL-expressing cells with high staining intensity.
Immunohistochemistry for Ki67 and PECAM

Ki67 is a cell cycle-associated nucleoprotein antigen that serves as a marker of proliferation during the active cell cycle phases $\left(\mathrm{G}_{1}, \mathrm{~S}, \mathrm{G}_{2}\right.$, and mitosis) [15]. An increase in Ki67 staining has been reported to correlate directly with activation of the flat granulosa cells of primordial follicles to cuboidal granulosa cells in primary follicles as well as with follicular growth.

PECAM-1 (CD31) is a member of the cell adhesion superfamily of molecules [19-22]. It is encoded by the PECAM-1 gene located on chromosome 17, and expressed in endothelial cells and most blood cells. PECAM molecules function in intracellular junctions of confluent vascular beds of endothelial cells. The protein is essential for maintaining blood-barrier control during venous white-cell migration and disposing of aging neurotrophils. It serves as a marker of neovascularization in mammalian ovaries [23] and is currently the marker most often used to identify changes in blood supply after grafting.

The procedures for follicular Ki67 staining have been described by us previously [15], and the procedure for graft PECAM immunostaining has been described by others [24]. Sections were incubated for one $\mathrm{h}$ with a rabbit polyclonal anti-Ki67 antibody (1:10 and 1:30, sc-15402, Santa Cruz Biotechnology, Santa Cruz, CA, USA). We have extensive experience with immunohistochemistry and often use two concentrations of primary antibodies, as the concentration that yields the best staining results varies between them [15]. The sections were similarly incubated with an anti-PECAM antibody (1:250, sc-8306, Santa Cruz Biotechnology). For the negative control, we used a normal rabbit IgG antibody (sc2027, Santa Cruz Biotechnology) diluted to the same concentrations as the primary antibody. For a positive control for Ki67, we used a section of a human tonsil (Cell Marque, Rocklin, CA, USA) and for PECAM, a section of a human kidney (described as a positive control by IHCWORLD, Life Science Products \& Services, Woodstock, MD, USA; see: http://www.ihcworld.com/_protocols/antibody_protocols/ cd31_pharmingen.htm).

The controls for IMH were according to the Histochemical Society's standards of practice [25]. The use of the renal tissue was approved by the local institutional ethics committee and an informed consent was obtained from the patient. Solutions from a Dako EnVision System, horseradish peroxidase-3amino-9-ethylcarbazole (AEC) kit (Dako Corporation, Glostrup, Denmark) were used for immunostaining: redbrown AEC staining indicated Ki67 or PECAM expression. We defined a follicle as being positively stained for Ki67 if at least one of its granulosa cells expressed Ki67 [15]. Two samples for Ki67 and two samples for PECAM from each patient in all the study groups underwent both staining procedures and were analyzed. 
PECAM staining intensity was graded on a 4-point scale similar to that used for the TUNEL staining and previously for VEGF-A [12,13] according to intensity: $0=$ no PECAM staining, $1=$ PECAM-expressing cells with very low intensity, $2=$ PECAM-expressing cells with medium staining intensity, and $3=$ PECAM-expressing cells with high staining intensity.

\section{Statistical analysis}

The slides from all the procedures were viewed independently and blindly by two of the authors (R.A. and N.F.), and the observations gave similar results. Data were analyzed by analysis of variance, chi-square test, and Fisher's exact test, as required. $P$ values less than 0.05 were considered statistically significant.

\section{Results}

The group characteristics and the results of treatment are shown in Tables 1 and 2.

Graft recovery rates ranged from 75 to $94 \%$, with no significant difference among the groups. Most tissue samples from the vitrified and frozen-thawed tissue alone were partially absorbed and shrunk. Figure 2 shows representative ovarian samples.

Follicle number was limited in the recovered grafts (Tables 1 and 2). The ungrafted fresh, frozen, and vitrified controls were from the same patients from which tissue was grafted after freezing. The number of atretic follicles (Tables 1 and 2; Fig. 3) was significantly higher after vitrification (group A), vitrification + improvement protocol (group B), and slow-freezing (group C) than that in fresh ungrafted, vitrified ungrafted, slow-frozen ungrafted, and after slow-freezing + improvement protocol (group D) (all differences, $p<0.0001$ ). However, group D specimens had more atretic follicles than those in the fresh ungrafted $(p<0.0001)$, vitrified ungrafted $(p<0.0001)$, and slow-frozen ungrafted $(p=0.03)$ controls. There were only a few atretic follicles in the ungrafted fresh/warmed/thawed specimens.

Apoptosis levels are shown in Fig. 4. A trend of an increase (with some significant differences) in stroma apoptosis was noted in the two vitrification groups (groups A and B). Apoptosis levels were higher in the vitrification group (group A) than those in the fresh and slow-frozen ungrafted groups ( $p=0.01$ for both). Apoptosis levels were also higher in the vitrification + improvement protocol group (group B) than those in the fresh ungrafted controls $(p=0.0009)$, slow-frozen $(p=0.0009)$, vitrified ungrafted $(p=0.01)$, and slow-frozen grafted group (group $\mathrm{C}, p=0.04$ ) and the slow-frozen group with the improvement protocol (group $\mathrm{D}, p=0.002$ ). It is
Fig. 2 Representative photographs of ungrafted controls and grafted samples. a Section of slow-frozen/thawed ungrafted ovarian sample from a 16-year-old girl (patient 2, as numbered in Tables 1 and 2). Note the primordial-primary follicle (arrow). Hematoxylin and eosin, original magnification $\times 400$. b Section of vitrified/warmed ungrafted ovarian sample from an 18-year-old woman (patient 4 , as numbered in Tables 1 and 2). Note the numerous primordial and primary follicles. Hematoxylin and eosin, original magnification $\times 400$. $\mathbf{c}$ Section of grafted ovarian sample from a 21-year-old woman after slow-freezing + the improvement protocol (group D) (patient 6, as numbered in Tables 1 and 2). Note the secondary and primary follicles (arrows) and the red-brown Ki67 staining in the granulosa cells and the oocytes. Original magnification $\times 400$. Note the negative control at the upper right hand side: a blue-stained (hematoxylin) secondary follicle without any traces of red-brown color and the positive control at the bottom left hand side with red-brown staining (arrows) between blue (hematoxylin) background staining. d Section of grafted ovarian sample from a 19-year-old woman after vitrification/warming + the improvement protocol (group C) (patient 5, as numbered in Tables 1 and 2). Note the atretic antral follicle. Hematoxylin and eosin, original magnification $\times 400$. e Section of a slow-frozen/thawed ovarian sample from the same patient as in panel a. Note the overall blue staining and lack of brown TUNEL staining, indicating lack of apoptosis. Original magnification $\times 400$. Note the positive control at the bottom left hand side of positively stained (red-brown) epithelium cells, a specimen from a rat mammary gland lobule taken 4 days after weaning. The negative control at the upper left hand side: blue-stained (hematoxylin) primordial follicle without any traces of redbrown. $\mathbf{f}$ Section of a grafted ovarian sample from the same patient as in panel $\mathbf{d}$ after vitrification/warming + improvement protocol. Note the brown TUNEL staining in the stroma cells and the follicle (arrows), indicating apoptosis. Original magnification $\times 400$. The positive control for TUNEL is shown in panel e. $\mathbf{g}$ Section of a grafted ovarian sample from the same patient as in panel $\mathbf{c}$ after slow-freezing/thawing + improvement protocol. Note the peripheral red-brown PECAM staining pattern in the graft's border. Original magnification $\times 400$. The negative control is at the upper right hand side, blue (hematoxylin)-stained stroma cells without any traces of red-brown. The positive control for PECAM is shown in panel $\mathbf{h}$. $\mathbf{h}$ Section of grafted ovarian sample from the same patient as in panels $\mathbf{c}$ and $\mathbf{g}$ after vitrification/warming. Note the scattered red-brown PECAM staining throughout the whole graft. Original magnification $\times 400$. Note the negative control at the upper right hand side, blue (hematoxylin)-stained stroma cells without any traces of red-brown, and the positive control at the lower left hand side with strong red-brown staining within blue background staining

noteworthy that differences were also higher although only close to significance in the slow-frozen grafted (group $\mathrm{C}, p>0.07)$ compared with those in the slow frozen grafted + improvement protocol (group D). A trend toward apoptosis reduction was noted in the group D (Figs. 2e, f and 4). It is noteworthy that we observed only 75 follicles in all the TUNEL-stained follicles; out of which only, eight $(\sim 11 \%)$ were very weakly attained for apoptosis (grade $0-1)$. All the follicles including the apoptotic ones were unevenly distributed in all the groups including the ungrafted controls.

Specific patterns of vascularization and PECAM expression correlated with type of treatment and follicle survival. In group $\mathrm{D}$, there was a peripheral as well as a diffuse (scattered throughout the specimen) patterns of PECAM staining (Figs. $2 \mathrm{~g}$ and 5), and more nonatretic 

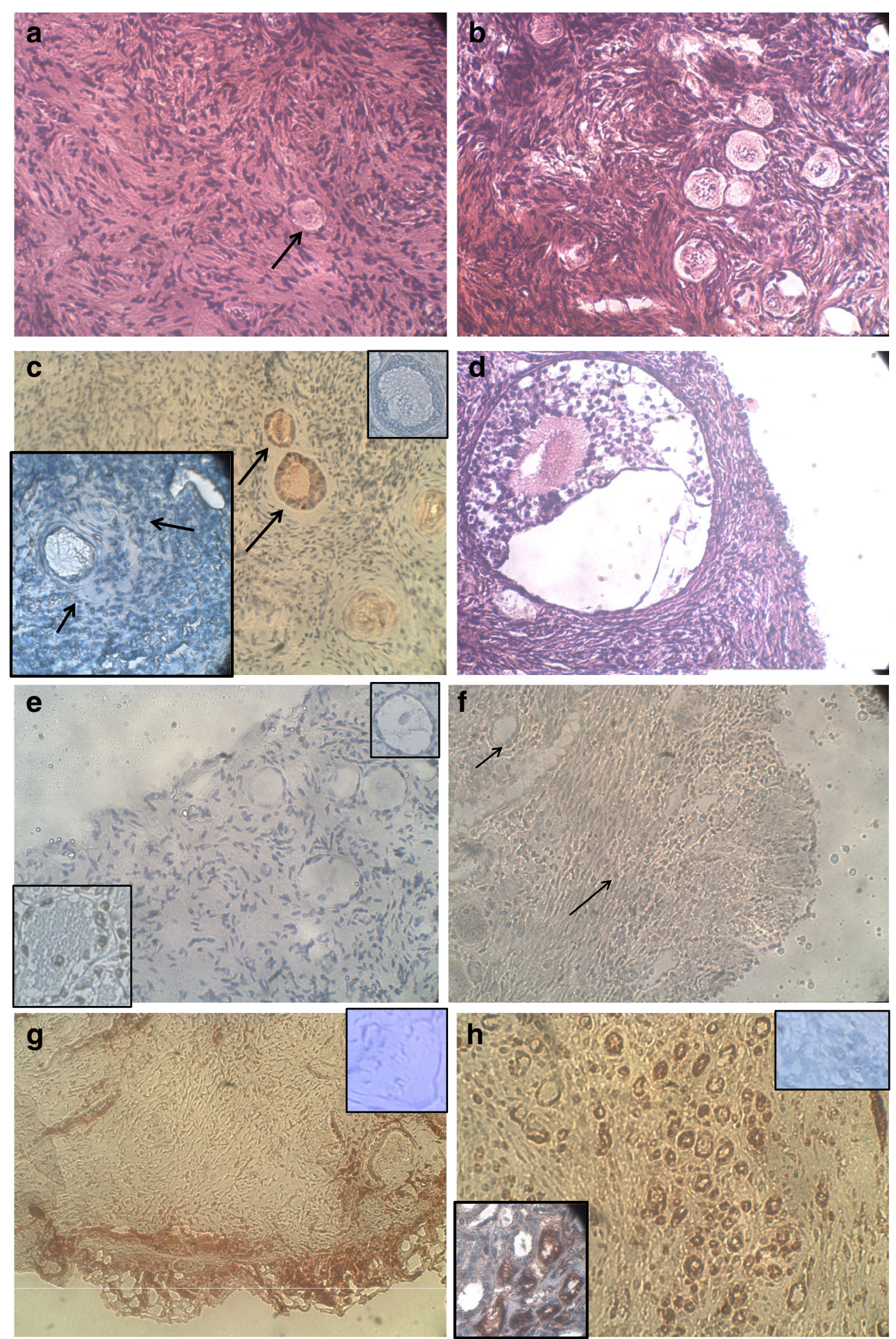

follicles were identified. In groups A-C, PECAM staining had diffuse pattern only (Fig. 2h). Additionally, specimens from groups A, C, and D had more blood vessels in the graft center and fewer follicles, whereas in group B specimens (vitrification + improvement protocol) not only was there no reduction in atretic follicles, but the PECAM staining pattern was similar to that of groups A and C.
The fresh ungrafted specimens and group D showed diffuse as well as peripheral staining pattern (the differences in the peripheral staining intensities were not significant), and the follicles were mostly morphologically normal and nonatretic (Fig. 5a, b).

Comparison of the diffuse staining intensities revealed significantly higher intensity in the vitrification-only 


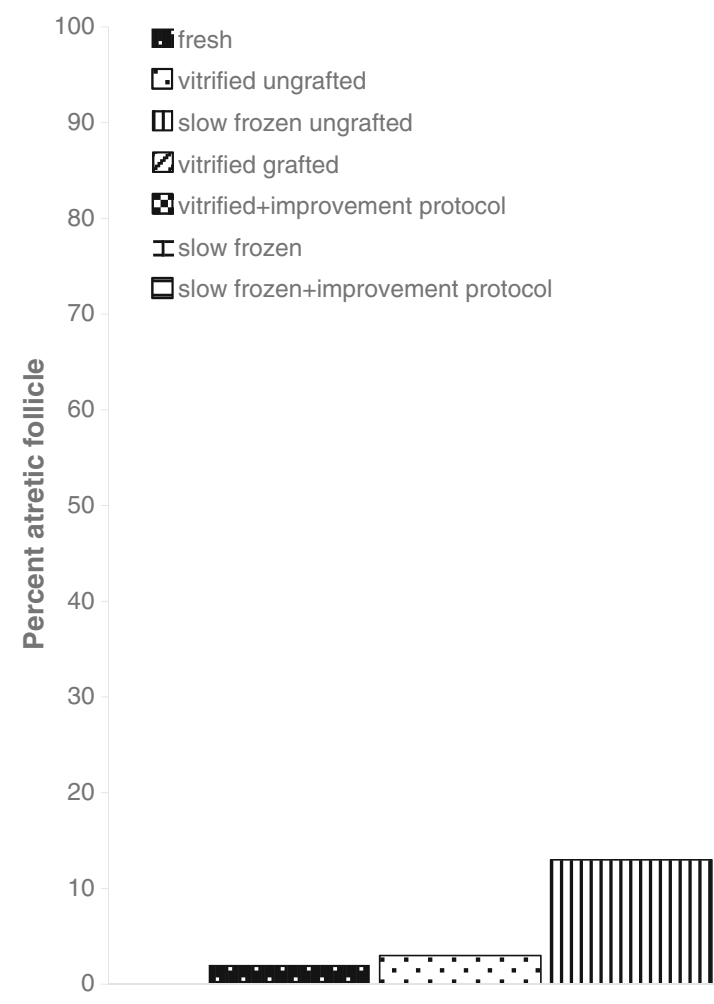

Fig. 3 Percents of atretic follicles in all the study groups The $x$ axis represents the four experimental groups and ungrafted fresh, vitrified/ warmed, and slow-frozen/thawed controls. The $y$ axis represents the percent of atretic follicles. Black bar with white dots = ungrafted fresh controls. White bar with black dots = ungrafted vitrified/warmed controls. Black and white perpendicular line bar = ungrafted slow-frozen/thawed controls. Black and white diagonal lines $=$ grafted vitrified $/$ warmed samples (group A). Black and white square bar = grafted vitrified/warmed samples + the improvement protocol (group B). Black and white brick

group (group A) than that in the slow-frozen samples (group $\mathrm{C}, p=0.01$ ); in the vitrification + improvement protocol group (group B) than that in the slow-frozen ungrafted $(p=0.006)$, the vitrified ungrafted $(p=$ 0.015 ), and the slow-frozen grafted group (group C, $p=0.0002$ ); and that in the slow-frozen group + improvement protocol (group D) than that in the vitrified + improvement protocol $(p=0.01)$. PECAM staining was detected in all blood vessels. Negative controls for PECAM stained only with blue hematoxylin and the positive controls stained with red-brown AEC staining (Fig. 2g, h).

Ki67 staining (Fig. 2c) in granulosa cells of morphologically normal follicles was detected in the fresh and frozen/thawed/warmed controls and grafted tissues. A total of 106 follicles were counted in all Ki67-stained sections; $80.2 \%$ stained positive for Ki67, with no significant differences among the groups. Negative controls for Ki67 stained only with blue hematoxylin (Fig. 2c) and the positive controls stained with red-brown AEC staining.

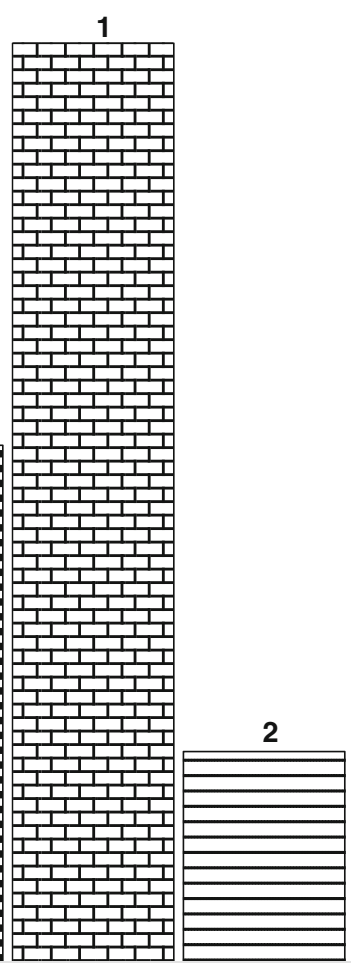

Groups

bar $=$ grafted slow-frozen/thawed samples (group C). Black and white parallel line bar $=$ grafted slow-frozen/thawed samples + the improvement protocol (group D). Superscript number one $\left({ }^{l}\right)$ indicates significantly higher than the fresh, vitrified, and slow-frozen ungrafted samples (Table 1) and grafted samples after slow-freezing + improvement protocol (group D) $(p<0.0001)$. Superscript number two $\left({ }^{2}\right)$ indicates significantly higher than the fresh $(p<0.0001)$, slow-frozen $(p=0.03)$, and vitrified $(p<0.0001)$ ungrafted controls

\section{Discussion}

In the present study, morphologically normal follicles were identified in all the ungrafted fresh, vitrified/warmed, or slow-frozen/thawed samples, with a high graft recovery rate after transplantation. The grafted specimens had fewer follicles than the control ungrafted fresh/warmed/thawed samples. The number of atretic follicles was significantly higher in the grafted specimens after vitrification with/without the improvement protocol and slow-freezing only (groups A-C) than in the grafted specimens after slow-freezing + the improvement protocol (group D). Stroma cell apoptosis levels were significantly higher in the specimens grafted after vitrification, without the improvement protocol (group A) than in fresh ungrafted and slow-frozen grafted samples. They were also higher after vitrification + improvement protocol (group B) than in fresh, vitrified and slow frozen ungrafted controls and in samples grafted after slow-freezing with/without the improvement protocol (groups C and D). We identified two staining patterns for PECAM in the grafted groups: peripheral 


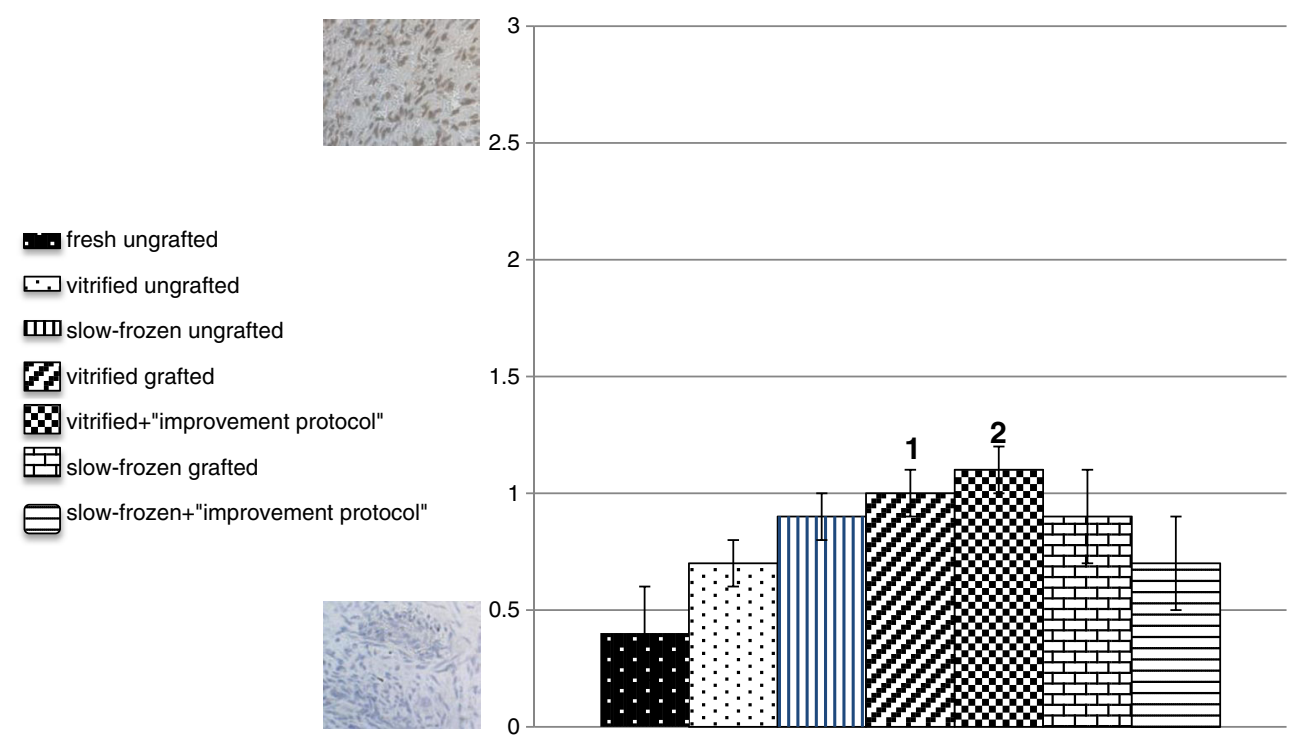

Fig. 4 Apoptosis expression in the ungrafted and grafted samples. Results are presented as mean $\pm \mathrm{SD}$. The $x$ axis represents the four experimental groups and ungrafted fresh, vitrified/warmed, and slowfrozen/thawed controls. The $y$ axis represents the level of TUNEL staining. Apoptosis (TUNEL) levels were graded according to intensity: $0=$ no apoptosis, $1=$ low staining intensity, $2=$ medium staining intensity, and 3 = high staining intensity. Black bar with white dots = ungrafted fresh controls. White bar with black dots $=$ ungrafted vitrified $/$ warmed controls. Black and white perpendicular line bar = ungrafted slow-frozen/thawed controls. Black and white diagonal lines = grafted vitrified/ warmed samples (group A). Black and white square bar=grafted

as well as diffuse in the group D, with more nonatretic follicles, and diffuse only in all the other grafted groups, with more blood vessels at the graft center and fewer follicles. It is noteworthy, however, that a peripheral as well as diffuse staining pattern was also identified in the fresh ungrafted controls. The diffuse staining intensity was significantly higher in the two vitrification groups (groups A and B) than that in the slowfrozen-only group (group C) and the vitrified/slow-frozen ungrafted controls. The diffuse staining pattern was also significantly higher in group D than group B. All the morphologically normal follicles were positive for Ki67 staining in the granulosa cells. Thus, grafting slow-frozen tissue combined with the improvement protocol yielded the best results in terms of the reduction in atretic follicles and stroma cell apoptosis, and the improvement protocol did not promote better outcomes after implantation of vitrified/warmed grafts. We also could not show a benefit of vitrification alone over slowfreezing.

Indeed, studies have shown that systemic administration of VEGF-A might lead to dangerous side effects and that high levels of local VEGF-A can induce development of damaged blood vessels [26]. However, to the best of our understanding, topical low-dose VEGF-A treatment does not cause side effects in the hosts, partly because of the short half-life (30 min) of VEGF-A in plasma [27]. vitrified/warmed samples + the improvement protocol (group B). Black and white brick bar= grafted slow-frozen/thawed samples (group C). Black and white parallel line bar $=$ grafted slow-frozen/thawed samples + the improvement protocol (group D). Superscript number one $\left({ }^{l}\right)$ indicates significantly higher than the fresh ungrafted $(p=0.01)$ and slowfrozen grafted $(p=0.01)$ groups. Superscript number two $\left({ }^{2}\right)$ indicates significantly higher than the fresh $(p=0.0009)$, slow-frozen $(p=$ $0.0009)$, and vitrified $(p=0.01)$ ungrafted samples, the samples grafted after slow-freezing only $(p=0.04)$, and the samples grafted after slowfreezing + improvement protocol $(p=0.002)$

The low number of surviving follicles in the grafted tissue could suggest accelerated follicular loss after transplantation. However, because of the uneven distribution of follicles in the human ovary as well as the small size of our grafted specimens, we may have inadvertently transplanted slices with no or only a few follicles $[1,12,28]$. Indeed, in three specimens, there were more follicles in the grafted thawed/warmed tissue than in the ungrafted thawed/warmed control tissue. The limited number of recovered follicles together with lack of significant differences among the groups in Ki67 staining precludes conclusions that follicular activation occurred after the short-term grafting or that there was "burn-out" of primordial follicles, especially in the thin-sliced vitrified tissue, as described for implanted bovine tissue [14]. Alternatively, it is also possible that we did not see follicular activation and burn-out because we grafted the tissue for longer than reported in the earlier study [14]. Because of the difficulties in obtaining human ovarian tissue for research, we use what is available, and patient ages are often not exactly in the same range. This might have contributed to the high standard deviations in the number of follicles per patient.

In our previous study [13], grafts transplanted after slowfreezing/thawing + the improvement protocol had no atretic follicles at all, whereas in identically treated tissue in the present study (group D), 22\% of the follicles were atretic. This 

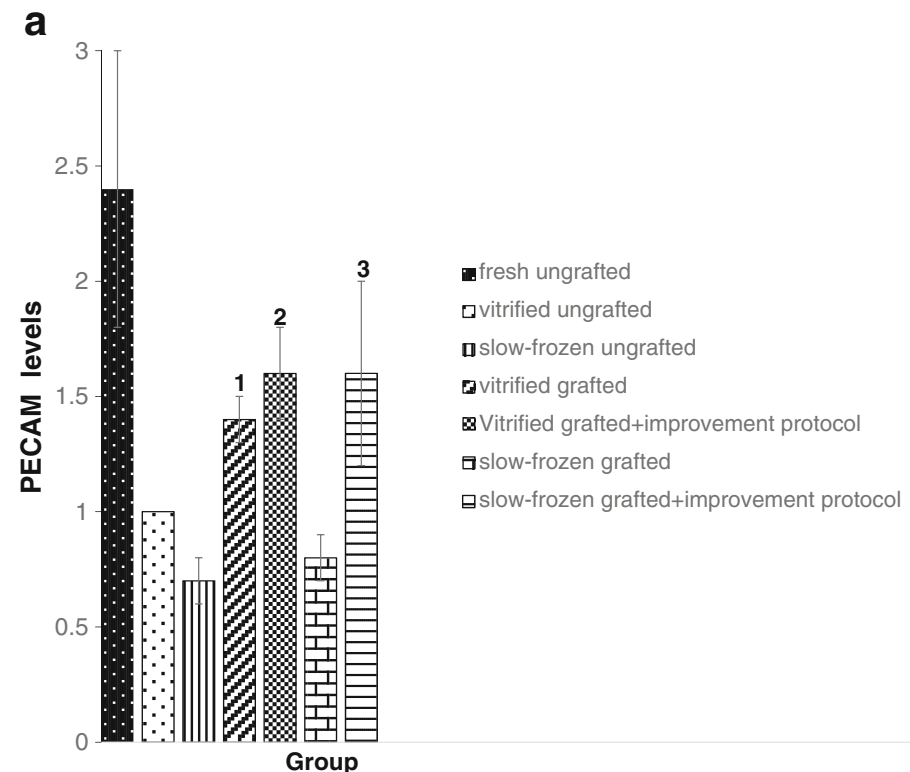

b

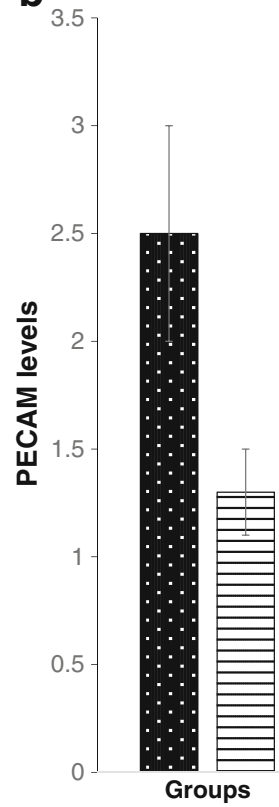

fresh ungrafted

घslow-frozen grafted

Fig. 5 PECAM expression in the ungrafted and grafted samples. a Diffuse PECAM expression in the ungrafted and grafted samples. Results are presented as mean $\pm \mathrm{SD}$. The $x$ axis represents the four experimental groups and ungrafted fresh, vitrified/warmed, and slowfrozen/thawed controls. The $y$ axis represents the level of PECAM staining. PECAM levels were graded according to intensity: $0=$ no PECAM staining, $1=$ low staining intensity, $2=$ medium staining intensity, and $3=$ high staining intensity. Black bar with white dots= fresh ungrafted controls with diffuse pattern. White bar with black dots $=$ vitrified $/$ warmed ungrafted controls with diffuse pattern. Black perpendicular line bar = ungrafted slow-frozen controls with diffuse pattern. Black diagonal line bar $=$ grafted vitrified/warmed samples (group A) with diffuse pattern. Black and white square bar=grafted vitrified/ warmed samples + improvement protocol (group B) with diffuse pattern.

difference might be explained by the use of more samples per group in the present study. Group D had fewer atretic follicles
Black and white brick bar = grafted slow-frozen/thawed samples (group C) with diffuse pattern. Black and white dark parallel line bar = grafted slow-frozen/thawed samples + the improvement protocol (group D) with diffuse pattern. Superscript number one $\left({ }^{l}\right)$ indicates significantly higher than the slow-frozen grafted $(p=0.01)$. Superscript number two $\left({ }^{2}\right)$ significantly higher than the slow-frozen $(p=0.006)$, vitrified $(p=0.015)$, ungrafted, and the samples grafted after slow-freezing $(p=0.0002)$ ungrafted. Superscript number three $\left({ }^{3}\right)$ indicates significantly higher than the grafted after vitrification + improvement protocol $(p=0.01) \mathbf{b}$ Peripheral PECAM expression in the fresh ungrafted and grafted slowfrozen/thawed samples t the improvement protocol (group D). Black bar with white dots $=$ fresh ungrafted fresh controls. Black and white dark parallel line bar=grafted slow-frozen/thawed samples + the improvement protocol (group D).

than in the other groups, but the number was still higher than that in the ungrafted slow-frozen control specimens. The 
increased percentage of atretic follicles in all the grafted groups relative to the ungrafted specimens may be attributed to follicular damage due to slow neovascularization. In addition, it is possible that needle-immersed vitrification causes long-term tissue/follicular damage that can be identified after grafting, but not immediately after short-term thawing/ warming. This might be explained by intracellular follicular changes that cannot be identified by light microscopy before grafting.

Our identification of stroma cell apoptosis in the grafts might indicate that nonapoptotic cells are important for graft implantation and survival. The highest apoptosis rates were detected after vitrification/warming with the improvement protocol, and the lowest rates, after slow-freezing with the improvement protocol, similar to our previous study [13].

The difference in the PECAM staining pattern between the grafted specimens in group D and that in the other grafted groups can be explained by differences in the rate of neovascularization [13]. It has been shown that both host and graft vessels contribute to revascularization of human ovarian tissue implanted in a murine hosts [29]: up to day 10 , neovascularization occurred mostly in the periphery, while thereafter, there was perfusion of mostly human blood vessels into the graft center. In our study, the identical staining pattern in the group $\mathrm{D}$ and the fresh ungrafted controls might indicate proper vascularization in the intact ovary, which is lost after slowfreezing alone. A similar post-grafting staining pattern was identified for VEGF-A in our earlier study in experiments identical to those in the group D [13]. Diffuse staining intensity was significantly higher in the two vitrification groups than that in the slow-frozen groups and the frozen-ungrafted controls, probably as a result of improper graft neovascularization which in turn promoted post-transplantation follicular loss. It is noteworthy that others [23] identified no PECAM staining before implantation and did not find any difference between PECAM staining after grafting vitrified or slowfrozen human tissue, possibly because of the use of a different vitrification method.

Little is known about the developmental capability of oocytes derived from pediatric patients. The report of a livebirth achieved after ovarian grafting of tissue cryostored at adolescence [30] is encouraging. In the present study, only two patients (ages 13 and 16 years) were adolescents. A previous study showed an increase in abnormal follicles in ovarian samples from prepubertal girls, with very little development in culture [31], but our long experience with ovarian tissue from pediatric patients does not support these findings for preantral follicles, either in histological sections or after culture or grafting $[12,13,15,32]$. The vitrification-only group included three additional patients that were the eldest in the study (ages 22, 29, and 31 years). However, there were no obvious differences in the number of follicles or of atretic follicles between the ungrafted and grafted vitrified specimens, and the ungrafted specimens from the 29-yearold patient were among those with the highest follicular counts among the ungrafted controls. Moreover, low numbers of recovered follicles were identified also in tissue from the three youngest patients (ages 13, 16, and 18 years).

Various studies have compared slow-freezing with vitrification in human ovarian tissue, but the results are so far inconclusive $[5-9,23,24,33,34]$. The inconsistencies among the studies may be explained by a lack of uniformity in the vitrification methods or the type or concentration of cryoprotectant used [5]. Some studies showed a benefit for vitrification [6], mainly in terms of stroma cell preservation. By contrast, in the present study, we did not find that vitrification decreased stroma cell apoptosis. Others reported that slow-freezing was associated with less follicular damage, fewer free radicals, and less apoptosis than vitrification [24], and one study found that neovascularization was similar for both freezing procedures after grafting, but the percent of grafted developing follicles was higher with slow-freezing [23]. These results are partially in line with ours, which showed better outcome for grafting after slow-freezing + the improvement protocol. One potential explanation is that vitrification is not a suitable method for human ovarian tissue, as these samples are very dense with small pores that do not allow proper penetration of the cryoprotectant solution in the short equilibration period. Another possibility is that our lack of experience with vitrification might explain the unsuccessful results compared to the other groups (3-9), although this seems very unlikely given that our group has many years of experience handling only human tissue $[1,12,13,15,32]$.

It is also possible that we might have obtained better results if we had applied different vitrification methods [5] or utilized devices for thin slicing of human ovarian tissue [7, 35] rather than slicing manually. Further studies are still warranted to determine the optimal freezing/thawing/grafting protocol for human ovarian tissue and to determine if surviving follicles undergo activation and further development after longer grafting periods.

Acknowledgments The authors are indebted to Ms. G. Ganzach from the Editorial Board of Rabin Medical Center for the English editing. The authors are also grateful to our laboratory technician Carmela Felz for the histological sections.

Compliance with ethical standards The study was approved by the local institutional ethics committee. Informed consent to donate tissue for the present study was obtained from the patients or the parents of minors.

\section{References}

1. Feigin E, Freud E, Fisch B, Orvieto R, Kravarusic D, Avrahami G, et al. Fertility preservation in female adolescents with malignancies. In: Moorland MT, editor. Cancer in female adolescents. Hauppauge: Nova; 2008. p. 103-38.

2. Jensen AK, Macklon KT, Fedder J, Ernst E, Humaidan P, Andersen CY. 86 successful births and 9 ongoing pregnancies worldwide in 
women transplanted with frozen-thawed ovarian tissue: focus on birth and perinatal outcome in 40 of these children. J Assist Reprod Genet. 2016. doi:10.1007/s10815-016-0843-9.

3. Kawamura K, Cheng Y, Suzuki N, Deguchi M, Sato Y, Takae S, et al. Hippo signaling disruption and Akt stimulation of ovarian follicles for infertility treatment. Proc Natl Acad Sci U S A. 2013;110:17474-4749.

4. Suzuki N, Yoshioka N, Takae S, Sugishita Y, Tamura M, Hashimoto M, et al. Successful fertility preservation following ovarian tissue vitrification in patients with primary ovarian insufficiency. Hum Reprod. 2015;30:608-15.

5. Amorim CA, Curaba M, Van Langendonckt A, Dolmans MM, Donnez J. Vitrification as an alternative means of cryopreserving ovarian tissue. Reprod Biomed Online. 2011;23:160-86.

6. Wang Y, Xiao Z, Li L, Fan W, Li SW. Novel needle immersed vitrification: a practical and convenient method with potential advantages in mouse and human ovarian tissue cryopreservation. Hum Reprod. 2008;23:2256-65.

7. Kagawa N, Silber S, Kuwayama M. Successful vitrification of bovine and human ovarian tissue. Reprod Biomed Online. 2009;18:568-77.

8. Keros V, Xella S, Hultenby K, Pettersson K, Sheikhi M, Volpe A, et al. Vitrification versus controlled-rate freezing in cryopreservation of human ovarian tissue. Hum Reprod. 2009;24:1670-83.

9. Xiao Z, Wang Y, Li L, Luo S, Li SW. Needle immersed vitrification can lower the concentration of cryoprotectant in human ovarian tissue cryopreservation. Fertil Steril. 2010;94:2323-8.

10. Amorim CA, Jacobs S, Devireddy RV, Van Langendonckt A, Vanacker J, Jaeger J, et al. Successful vitrification and autografting of baboon (Papio anubis) ovarian tissue. Hum Reprod. 2013;28:2146-56.

11. Dolmans MM, Binda JF, Jacobs S, Dehoux JP, Squifflet JL, Ambroise J, et al. Impact of cryopreservation technique and vascular bed on ovarian tissue transplantation in cynomolgus monkeys. J Assist Reprod Genet. 2015;32:1251-62.

12. Abir R, Fisch B, Jessel S, Felz C, Ben-Haroush A, Orvieto R. Improving posttransplantation survival of human ovarian tissue by treating the host and graft. Fertil Steril. 2011;95:1205-10.

13. Friedman O, Orvieto R, Fisch B, Felz C, Freud E, Ben-Haroush A, et al. Possible improvements in human ovarian grafting by various host and graft treatments. Hum Reprod. 2012;27:474-82.

14. Gavish Z, Peer G, Roness H, Cohen Y, Meriow D. Follicle activation and 'burn-out' contribute to post-transplantation follicle loss in ovarian tissue grafts: the effect of graft thickness. Hum Reprod. 2016;29:989-96.

15. Lerer-Serfaty G, Samara N, Fisch B, Shachar M, Kossover O, Seliktar D, et al. Attempted application of bioengineered/ biosynthetic supporting matrices with phosphatidylinositoltrisphosphate-enhancing substances to organ culture of human primordial follicles. J Assist Reprod Genet. 2013;30:1279-88.

16. Telfer EE, McLaughlin M, Ding C, Thong KJ. A two-step serumfree culture system supports development of human oocytes from primordial follicles in the presence of activin. Hum Reprod. 2008;23:1151-8.

17. Abir R, Biron-Shental T, Orvieto R, Garor R, Krissi H, Fisch B. Transplantation of frozen-thawed late-gestational-age human fetal ovaries into immunodeficient mice. Fertil Steril. 2009;92:770-7.

18. Gougeon A. Regulations of ovarian follicular development in primates: facts and hypotheses. Endocr Rev. 1996;17:121-54.
19. Gumina RJ, Kirschbaum NE, Rao PN, Kenny D, Warltier DC, Newman PJ, et al. The human PECAM1 gene maps to $17 \mathrm{q} 23$. Genomics. 1996;34:229-32.

20. Fraser HM. Regulation of the ovarian follicular vasculature. Reprod Biol Endocrinol. 2006;12:4-18.

21. Woodfin A, Voisin MB, Nourshargh S. PECAM-1: a multifunctional molecule in inflammation and vascular biology. Arterioscler Thromb Vasc Biol. 2007;27:2514-23.

22. Privratsky JR, Newman DK, Newman PJ. PECAM-1: conflicts of interest in inflammation. Life Sci. 2010;87:69-82.

23. Rahimi G, Isachenko V, Kreienberg R, Sauer H, Todorov P, Tawadros $\mathrm{S}$, et al. Re-vascularisation in human ovarian tissue after conventional freezing or vitrification and xenotransplantion. Eur $\mathrm{J}$ Obstet Gynecol Reprod Biol. 2010;149:63-7.

24. Isachenko V, Lapidus I, Isachenko E, Krivokharchenko A, Kreienberg R, Woriedh M, et al. Human ovarian tissue vitrification versus conventional freezing: morphological, endocrinological and molecular biological evaluation. Reproduction. 2009;138:319-27.

25. Hewitt SM, Baskin DG, Frevert CW, Stahl WL, Rosa-Molinar E. Controls for immunohistochemistry: the Histochemical Society's standards of practice for validation of immunohistochemical assays. J Histochem Cytochem. 2014;62:693-7.

26. Ehrbar M, Djonov VG, Schnell C, Tschanz SA, Martiny-Baron G, Schenk U, et al. Cell-demanded liberation of VEGF121 from fibrin implants induces local and controlled blood vessel growth. Circ Res. 2016;94:1124-32.

27. Tang J, Hua Y, Su J, Zhang P, Zhu X, Wu L, et al. Expression of VEGF and neural repair after alprostadil treatment in a rat model of schiatic nerve crush injury. Neurol India (Serial: Online). 2009;57:387-94.

28. Schmidt KL, Byskov AG, Nyboe-Andersen A, Müller J, Andersen Yding C. Density and distribution of primordial follicles in single pieces of cortex from 21 patients and in individual pieces of cortex from three entire human ovaries. Hum Reprod. 2003;18:1158-64.

29. Van Eyck A-S, Bouzin C, Feron O, Romeu L, Van Langendonckt A, Donnez J, et al. Both host and graft vessels contribute to revascularization of xenografted human ovarian tissue in a murine model. Fertil Steril. 2010;93:1676-85.

30. Demeestere I, Simon P, Dedeken L, Moffa F, Tsepelidis S, Branchet C, et al. Live birth after autograft of ovarian tissue cryopreserved during childhood. Hum Reprod. 2015;30:2107-9.

31. Anderson RA, McLaughlin M, Wallace WH, Albertini DF, Telfer EE. The immature human ovary shows loss of abnormal follicles and increasing follicle developmental competence through childhood and adolescence. Hum Reprod. 2014;29:97-106.

32. Lande Y, Fisch B, Tsur A, Farhi J, Prag-Rosenberg R, Ben-Haroush A, et al. Short term exposure of human ovarian follicles to cyclophosphamide metabolites seems to promote follicular activation in vitro. RBM Online. 2016;34:104-14.

33. Li YB, Zhou CQ, Yang GF, Wang Q, Dong Y. Modified vitrification method for cryopreservation of human ovarian tissues. Chin Med J. 2007;120:110-4.

34. Huang L, Mo Y, Wang W, Li Y, Zhang Q, Yang D. Cryopreservation of human ovarian tissue by solid-surface vitrification. Eur J Obstet Gynecol Reprod Biol. 2008;139:193-8.

35. Revel A, Laufer N, Ben Meir A, Lebovich M, Mitrani E. Microorgan ovarian transplantation enables pregnancy: a case report. Hum Reprod. 2011;26:1097-103. 